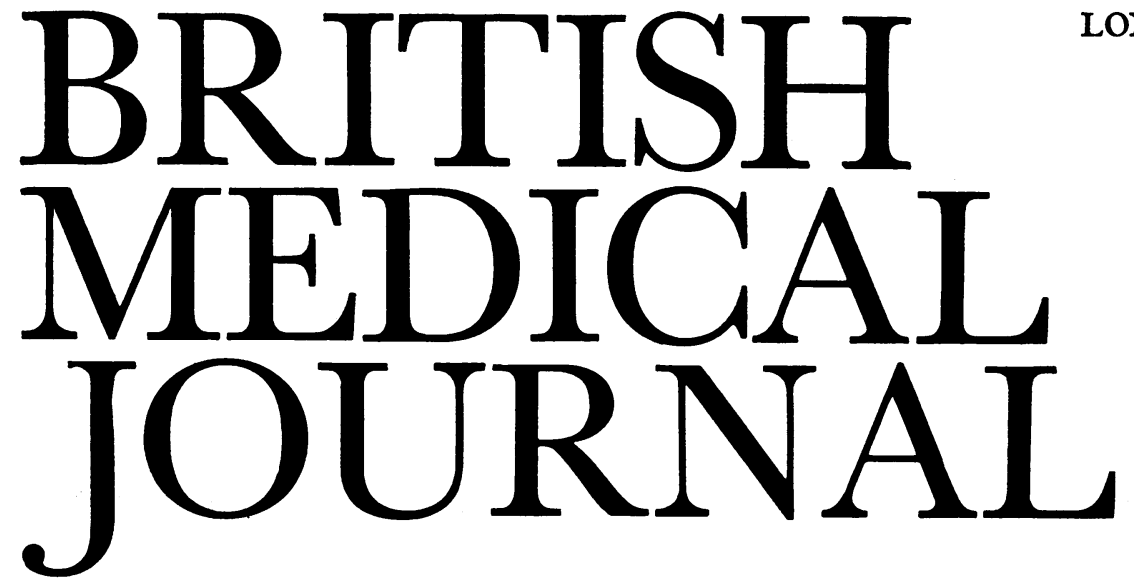

LONDON, SATURDAY 2 SEPTEMBER 1978

\title{
Modified neurosyphilis
}

The classical descriptions of tabes dorsalis, general paralysis of the insane (GPI), and meningovascular syphilis have been familiar to generations of medical students. Nevertheless, in their fully developed forms these diseases have become rarities in advanced countries. Though the incidence of neurosyphilis began to decline at the start of the century ${ }^{1}$ its present low level is probably due largely to effective treatment of early syphilis by penicillin and other antitreponemal drugs. Furthermore, Catterall ${ }^{2}$ has pointed out, the predicted rise in the number of cases of neurosyphilis resulting from untreated infections acquired during the second world war never materialised. Giving antibiotics for other diseases may have resulted in some patients with undiagnosed syphilis receiving treatment by chance, thereby preventing late complications. Some have argued, however, that this "prevention" is illusory, and that neurosyphilis still occurs, though often in a modified form with minimal symptoms and signs.

In any investigation of this hypothesis we must clearly define the diagnostic criteria. Hooshmand et al ${ }^{3}$ studied 241 patients in whom neurosyphilis had been diagnosed on the basis of a positive fluorescent treponemal antibody (absorbed) test (FTA-ABS) and ocular or neurological findings suggestive of neurosyphilis; or positive blood and cerebrospinal fluid FTA-ABS with over five polymorphonuclear leucocytes $/ \mathrm{mm}^{3}$ in the cerebrospinal fluid, with no evidence of bacterial or viral meningitis; or positive FTA-ABS results in blood and cerebrospinal fluid in patients with progressive neurological diseases in whom other aetiological factors had been excluded. An additional requirement in this last subgroup was that either the cerebrospinal fluid should show an increased leucocytosis or that the patient should improve clinically after treatment with penicillin. Focal or general seizures were the most common presenting symptoms, but others included poor vision and other ocular abnormalities, dizziness, and confusion after a cerebrovascular accident. Only a few patients showed psychiatric symptoms, but this may have been due to the type of referrals; while a few patients had the clinical picture of GPI, most were categorised under nonspecific diagnosis such as depression. Nearly half the patients in the study group had unrelated symptoms and neurosyphilis had been diagnosed only during routine investigation. Some clinical neurological abnormality was found in over threequarters of the patients. Reflex changes (particularly absent ankle reflexes) were the most frequent, though the least specific, but one-third of the patients had signs of posterior column lesions. In those attending for routine medical examination ophthalmic signs were the most common abnormality; these included pupillary changes, ptosis, uveitis, and choroidoretinitis. All the patients had positive blood FTA-ABS, but reagin tests were positive in only $117(48.8 \%)$; similar serological results were obtained in those specimens of cerebrospinal fluid examined, all being reactive in the FTA-ABS but only about one-half showing reagin.

Broadly comparable findings have been reported by Joyce-Clark and Molteno ${ }^{4}$ in a recent study of neurosyphilis in the Cape Peninsula, though many of their patients had been referred by ophthalmologists with suspicious ocular signs. They concluded that the most reliable and consistently helpful diagnostic features were abnormal pupillary reflexes, the blood FTA-ABS reaction, and raised concentrations of protein in the cerebrospinal fluid. Cerebrospinal fluid serology and the cell count were far less reliable markers. Included in the groups with "diagnostic" or "suspicious" signs were a subgroup of 13 patients with totally negative blood reactions, the diagnosis here depending on clinical signs and raised protein concentrations in the cerebrospinal fluid which declined after treatment.

Clearly, then, while classical cases of neurosyphilis still occur, we need to revise the clinical and laboratory criteria established in preantibiotic days. The diagnosis of neurosyphilis depends on a clinical assessment of the importance of symptoms and signs, and the results of serological tests and examination of the cerebrospinal fluid. In atypical cases none of these is pathognomonic. Only a few patients show classical diagnostic signs such as the Argyll Robertson pupil; many of the clinical abnormalities may just as well be due to other diseases.

Conflicting views ${ }^{34}$ have been expressed on the diagnostic importance of estimations of the protein concentration and the cell count in the cerebrospinal fluid. Serological tests are useful but not infallible. Reagin tests are too insensitive to be of value, and reliance must be placed on specific tests. ${ }^{5}$ Nevertheless, a positive blood FTA-ABS test may indicate totally inactive infection, and difficulties have been reported in diagnosing neurosyphilis in populations with a high proportion of seroreactors. ${ }^{6}$ In the cerebrospinal fluid a 
positive FTA-ABS test is invariably associated with a positive test in the serum (so to perform this test on the cerebrospinal fluid in patients with negative blood FTA-ABS tests is totally unproductive). A definitely positive result in the cerebrospinal fluid is certainly important (provided that contamination with blood during lumbar puncture is excluded), and Wilkinson ${ }^{7}$ reported that the FTA-ABS test on the cerebrospinal fluid was virtually always positive when the cell count and protein concentration were abnormal. He had reservations about using an unabsorbed FTA test on undiluted cerebrospinal fluid as a screening test, which are shared by Jaffe et $a l^{8}$ in a recent study. As a sole abnormal finding a reactive FTA-ABS in the cerebrospinal fluid may not necessarily indicate active neurosyphilis: the result may have persisted after antibiotic treatment for either syphilis or some other condition.

The diagnosis of neurosyphilis-now just as much as in the past-requires the critical evaluation of a series of findings and the criteria of Hooshman et $a l^{3}$ should be widely used. Atypical neurosyphilis may be commoner than is generally supposed. The widespread use of antibiotics for treating unrelated conditions may have led to some patients with unsuspected syphilis receiving suboptimal treatment. Unfortunately recent evidence ${ }^{910}$ shows that some recommended therapeutic regimens-especially those using benzathine penicillin-may give poor concentrations of penicillin in the cerebrospinal fluid. Possibly, therefore, even some patients treated for early syphilis in venereal disease clinics may later develop modified neurosyphilis.

${ }^{1}$ Martin, J P, British Medical fournal, 1972, 3, 159.

2 Catterall, R D, British fournal of Hospital Medicine, 1977, 17, 585.

${ }^{3}$ Hooshmand, H, Escobar, M R, and Kopf, S W, fournal of the American Medical Association, 1972, 219, 726.

4 Joyce-Clarke, N, and Molteno, A C B, South African Medical fournal, 1978, 53, 10.

5 John, J F, and Cuetter, A C, Southern Medical fournal, 1977, 70, 309.

6 Ashcroft, M T, et al, British fournal of Venereal Diseases, 1967, 43, 96.

7 Wilkinson, A E, British fournal of Venereal Diseases, 1973, 49, 346.

$8 \mathrm{Jaffe}, \mathrm{H} \mathrm{W}$, et al, Archives of Internal Medicine, 1978, 138, 252.

9 Tramont, E C, fournal of the American Medical Association, 1976, 236, 2206.

${ }^{10}$ Mohr, J A, et al, fournal of the American Medical Association, 1976, 236, 2208.

\section{Wheelchairs}

Providing wheelchairs for the disabled is the responsibility of DHSS artificial limb and appliance centres. The system has long been notorious for its delays, ${ }^{1}$ and a report from the Wolfson Centre in London ( $p$ 688) shows that delays of six months are still commonplace, even for the more frequently prescribed folding self-propelled chairs, such as the 8 and 8BL models.

These delays cause unacceptable hardship-particularly in patients with progressive disability, such as multiple sclerosis, and in children. Sometimes the delay is so long that when it arrives the wheelchair is unsuitable. Non-standard chairs, electrically propelled chairs, and special models such as the BEC or the Everest and Jennings chairs are often not delivered for nearly a year. When the order comes from a specialised wheelchair clinic such a delay is impossible to justify: usually the prescription is accurate and the paper work routine. General practitioners can now prescribe wheelchairs themselves, but they have to face the same problems.

One explanation could be that the DHSS is making small economies by slow ordering from the approved manufacturers rather than keeping a central stock of chairs. Yet with bulk buying and computer record keeping costs could surely be reduced and the service to the customer improved. While relatively few people are affected and lives are not at stake, this wheelchair famine is one source of misery and frustration that could surely be removed by a determined effort. Perhaps Mr David Ennals could look at it himself-he has, after all, recent personal experience of the value of a wheelchair in providing mobility.

${ }^{1}$ Holt, K S, Darcus, H, and Brand, H L, British Medical fournal, 1972, 4 , 651 .

\section{Adverse reactions to intravenous induction agents}

Forty-four years after thiopentone was introduced it still dominates intravenous induction of anaesthesia and provides the standard by which all drugs of similar purpose are judged. These include methohexitone, propanidid, Althesin, etomidate, and ketamine; neuroleptic agents such as droperidol; analgesics such as fentanyl; and benzodiazepines such as diazepam. Since the introduction of propanidid and more recently Althesin allergic or anaphylactic reactions have begun to cause concern. The common features include erythema, rashes, urticaria, oedema of the air passages, bronchospasm, and cardiovascular collapse.

Adverse reactions ${ }^{1}$ may be due to direct pharmacological release of histamine, to immune-mediated (type I Coombs and $\mathrm{Gell}^{2}$ ) mechanisms, or to activation of $\mathrm{C} 3$ complement leading to release of histamine. The first and third of these responses may occur at the first exposure to the drug and should therefore be described as idiosyncratic or anaphylactoid reactions. Mediators other than histamine may be concerned. ${ }^{3}{ }^{4}$ Nevertheless, most reactions to these drugs are not anaphylactoid: they are simple side effects or the effects of overdose in either normal individuals or in those who are intolerant to the drug and have exaggerated responses.

The incidence of anaphylactoid reactions appears to be increasing. ${ }^{5}$ Reviews published ${ }^{6-10}$ list 44 reactions to thiopentone, 10 to methohexitone, over 29 to propanidid (one fatal), 113 to Althesin (one fatal), one to ketamine, ${ }^{11}$ one to diazepam, ${ }^{12}$ but so far none to droperidol, fentanyl, or etomidate. The incidence of reactions to propanidid is higher than that to thiopentone, ${ }^{679}$ and fortunately the number of reactions to the barbiturates remains extremely low. Clarke et al ${ }^{9}$ estimated that the incidence of reactions to Althesin was between one in 14000 and one in 19000 . Nevertheless, other reports indicate an incidence of one in $900^{713}$ or one in $1900 .^{10}$

Neuroleptic drugs, ketamine, and diazepam have limited applications. ${ }^{6}$ They have a long action and their complications include increased muscle tone and severe respiratory depression with large doses of fentanyl and dissociative phenomena, hallucinations, and unpleasant dreams with ketamine.

Induction of anaesthesia with the remaining drugs may be complicated by cardiorespiratory depression, muscle movements, cough, hiccough, and laryngospasm. Althesin causes a relatively low incidence both of these motor phenomena and of respiratory depression and has a reputation for smooth induction of anaesthesia. Etomidate causes a high incidence of involuntary muscle movements (between $60 \%$ and $98 \%$ ), ${ }^{14} 15$ 